\title{
Versatile cryogen-free cryostat for the electromagnetic characterization of superconducting radiofrequency coils
}

Isabelle Saniour ${ }^{1 * \dagger}$ (D), Michel Geahel ${ }^{1,2,3 \dagger}$, Javier Briatico ${ }^{2}$, Cornelis J. van der Beek ${ }^{3,4}$, Georges Willoquet ${ }^{1}$, Laurène Jourdain ${ }^{1}$, Bertrand Baudouy ${ }^{5}$, Gilles Authelet ${ }^{5}$, Jean-Christophe Ginefri ${ }^{1}$, Luc Darrasse ${ }^{1}$ and Marie Poirier-Quinot ${ }^{1}$

\author{
* Correspondence: isabelle.saniour@ \\ universite-paris-saclay.fr \\ Isabelle Saniour and Michel Geahel \\ are Co-first authors. \\ ${ }^{\dagger}$ Isabelle Saniour and Michel Geahel \\ contributed equally to this work. \\ ${ }^{1}$ Université Paris-Saclay, CEA, CNRS, \\ Inserm, BioMaps, Orsay, France \\ Full list of author information is \\ available at the end of the article
}

\begin{abstract}
The use of high temperature superconducting (HTS) radio frequency (RF) coils in Magnetic Resonance Imaging (MRI) greatly improves the signal-to-noise ratio (SNR) in many biomedical applications and particularly in micro-MRI. However, a detailed understanding of the electrical behavior of HTS coils is important in order to optimize their performance through MR experiments. This paper presents a simple and versatile cryogen-free cryostat designed to characterize the RF properties of HTS coils prior to their use in MRI. The cryostat can be used at temperatures from $50 \mathrm{~K}$ to $300 \mathrm{~K}$, with a control precision of approximately $3 \mathrm{mK}$ at $70 \mathrm{~K}$, and can measure the RF electrical power transmitted to an HTS coil over a range from $1 \mu \mathrm{W}$ to $10 \mathrm{~W}$. The quality factor and resonance frequency of the tested HTS coil are determined as a function of the temperature and the power it dissipates. This cryostat also permits the dynamic adjustment of the coil resonance frequency via temperature control. Finally, this study demonstrates that the HTS coil takes less than $12 \mu \mathrm{s}$ to transit from the superconducting to the dissipative state, which is compatible with MRI requirements.
\end{abstract}

Keywords: Superconductivity, HTS coil, Cryostat, High sensitivity

\section{Introduction}

Magnetic resonance imaging (MRI) is a safe and non-invasive imaging technique that provides anatomical, structural and functional information. In general, radiofrequency (RF) volume transmit coils are used to generate a homogeneous spin excitation. The detection of the spin response is achieved by placing a surface receive coil close to the region of interest; surface coils have a higher local sensitivity than volume coils. The receive coil must be inactive when the transmit coil is active (i.e., during spin excitation) in order to prevent RF coupling between the two coils. This is usually obtained by using an active or passive decoupling circuit that is soldered on the coil, thus attenuating or shifting the resonance of the coil [1].

(c) The Author(s). licensee Springer on behalf of EPJ. 2020 Open Access This article is licensed under a Creative Commons Attribution 4.0 International License, which permits use, sharing, adaptation, distribution and reproduction in any medium or format, as long as you give appropriate credit to the original author(s) and the source, provide a link to the Creative Commons licence, and indicate if changes were made. The images or other third party material in this article are included in the article's Creative Commons licence, unless indicated otherwise in a credit line to the material. If material is not included in the article's Creative Commons licence and your intended use is not permitted by statutory regulation or exceeds the permitted use, you will need to obtain permission directly from the copyright holder. To view a copy of this licence, visit http://creativecommons.org/licenses/by/4.0/. 
In high spatial resolution MRI, the nuclear magnetic resonance (NMR) signal encoded in an elementary voxel is relatively weak because of the microscopic dimension of the voxel. This means that the RF surface coil used for signal detection has to be highly sensitive. One solution for this sensitivity-based limitation is to use high temperature superconducting (HTS) receiver coils at clinical field strength. This has been shown to greatly improve the signal-to-noise ratio (SNR) [2] and has been successfully used in micro-imaging applications $[3,4]$. The SNR improvement is attributable to both the small size of the coil and to the low resistance $(R)$ of the HTS material in the superconducting state at low cryogenic temperature. Its resistance is several orders of magnitude lower than that of conventional copper and results in a substantially lower noise level.

However, the standard decoupling techniques mentioned above cannot be used with HTS coils because the use of active or passive soldered components would entail significant additional losses and negate the advantages provided by the low resistance of the HTS material. This explains why previous works were conducted using HTS coils in both transmit and receive modes. Besides, HTS materials exhibit a nonlinear variation of their resistance as a function of the transmitted power. The use of HTS coil in transmit mode thus results in a non-uniform/controlled flip angle and makes impossible the production of quantitative images. It is therefore important to find dedicated strategies to inactivate/decouple the HTS coil during RF transmission.

One possible solution is to take advantage of the resistance variation as a function of the power transmitted to the HTS coil. The power that the HTS coil is exposed to during the RF transmission period (about $1 \mathrm{~W}$ ) far exceeds the power applied during the reception period (up to $10^{-6} \mathrm{~W}$ ). Shen $\mathrm{el} \mathrm{al}$. have found that the resistivity of superconducting materials presents a nonlinear behavior under the effect of RF power [5], but no general model to describe or predict the resistance variation of HTS coils over an extended range of temperature and power has been published to date [6].

Girard et al.'s [7] method to measure the quality factor $(Q)$ and the resonance frequency $\left(f_{0}\right)$ of an HTS coil makes it possible to predict coil resistance during RF transmission $[8,9]$. However, a dedicated set-up is required to ensure that measurements are accurate, reproducible and reliable enough to use resistance variation to activate the HTS coil during RF reception and inactivate it during RF transmission. This setup should also make it possible to measure the transition time from the superconducting to the dissipative state. This switching time has to be short compared to the few milliseconds observed in typical RF pulse durations.

There are different approaches to develop a dedicated cryostat to cool RF coils. Most cooling systems involve the direct immersion of the coil in liquid nitrogen (LN2) using for example Styrofoam vessels [10-14]. Some publications report the use of cold fingers made of solid materials with high thermal conductivity and low dielectric losses, such as sapphire or alumina [15-18]. Nevertheless, all of these approaches involve working with the cryogenic fluid at a fixed temperature defined by the saturation conditions at atmospheric pressure. To overcome this, Lambert et al. propose a technique in which users can vary the temperature of a LN2 cryostat by controlling the pressure of the nitrogen bath [19]. However, this method presents risks in terms of pressure hazards, and does not provide accurate and precise RF measurements due to the intrinsic instability of the LN2 pressure. Another means to control the temperature variation is to 
use closed-cycle cryocoolers such as Joule-Thomson (J-T) cryocoolers [20], GiffordMcMahon [21], pulse-tube [22] and Stirling cryogenerators [23] that allow to reach liquid nitrogen temperature without the use of cryogens. These closed-cycle cryocoolers are cryogen-free and most of them rely on a hermetically sealed volume of gas that is periodically compressed and expanded by a piston and a displacer.

This paper presents a custom-made, low-cost cryogenic system with an integrated radiofrequency characterization setup. It allows users to study the properties of superconducting materials as a function of temperature and RF power through contactless electrical measurements. The cooling source is based on the Stirling cryocooler, a compromise between cost and efficiency. This cryogen-free cryostat combines a dedicated cryogenic environment with an RF measurement setup, thus allowing for the simultaneous monitoring of temperature and the evaluation of $f_{0}$ and $Q$ prior to their use in MRI.

\section{Methods/experimental}

The experimental setup is composed of a vacuum chamber associated with a cryocooler, a pressure monitoring system, a temperature controller and an RF measurement system. A schematic diagram and a photograph of the cryostat are provided in Fig. 1a and $\mathrm{b}$, respectively.

\section{Cryogenic system}

The vacuum chamber is a sealed four-way cross (304 L, CF160) with a diameter of $152.4 \mathrm{~mm}$ and a height of $273 \mathrm{~mm}$ (Fig. 1b). The cold head is installed at the bottom of the cross; the coil under test is placed inside the vacuum chamber via a loadlock door with a transparent window, located at the top of the cross. Electrical and vacuum feedthroughs are placed on the sides of the cross as shown in Fig. 1a. A micrometric screwdriver is used to finely adjust the position of the measurement probe (see Fig. 1b). A turbo-molecular vacuum pump is connected to the evacuation part of the vacuum chamber. Dynamic pumping is used during the experiments so as to maintain a vacuum of about $10^{-5}$ mbar.

Cooling power is provided by a LSF 9340 cryocooler (THALES Cryogenics, Eindhoven, Netherlands). A copper plate is screwed onto the cold finger of the cryocooler to host the sapphire cylinder (height and diameter $=20 \mathrm{~mm}$ ) used to eliminate eddy currents that are induced in metallic sample holders when RF magnetic fields are present. To ensure thermal contact, indium foils are systematically placed at each interface i.e. between the cold finger and the copper plate and between the copper plate and the sapphire cylinder.

\section{Temperature monitoring system}

Two temperature sensors are used for temperature monitoring (see Fig. 1a). A PNP 2N5195 transistor is screwed to the copper plate hosting the sapphire cylinder. The emitter-base diode of this transistor is used as a temperature sensor. Connected as it is to the cooler drive electronics (provided by THALES cryogenics), the diode measures the temperature of the cold head using a custom-made Matlab interface. The temperature of the RF coil is probed with a flat Pt100-type Platinum resistor (RS Pro 
a)

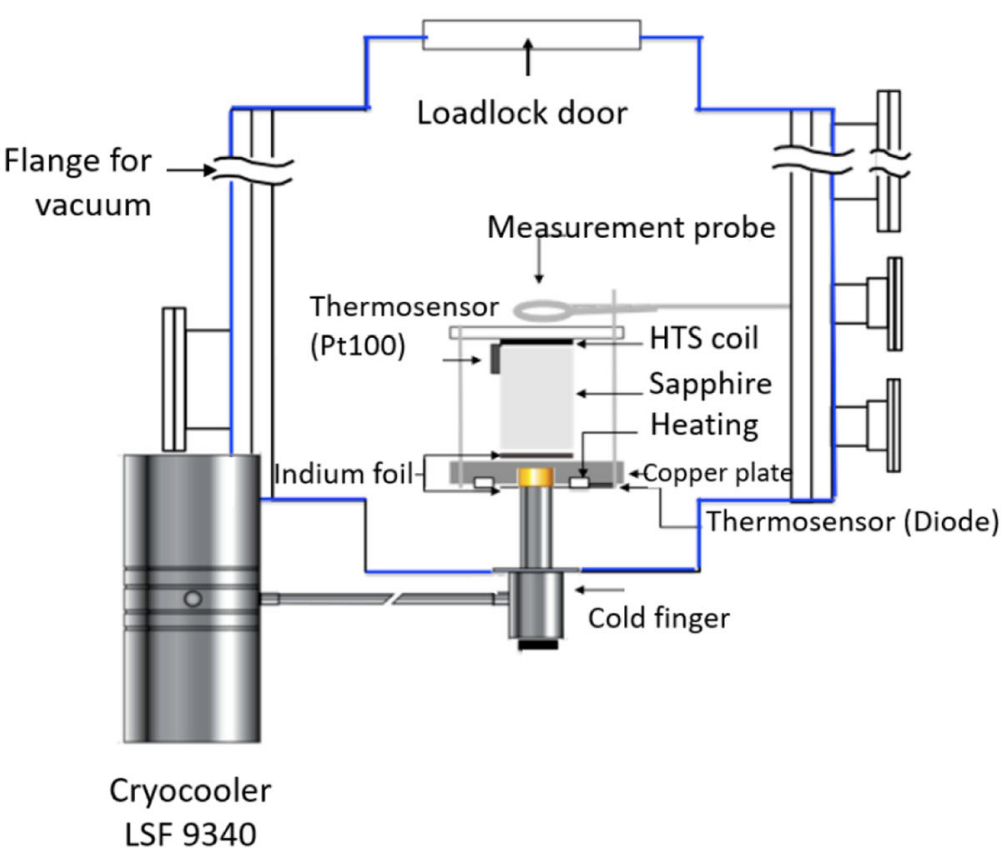

b)

Vacuum pump

Flange for

Vacuum

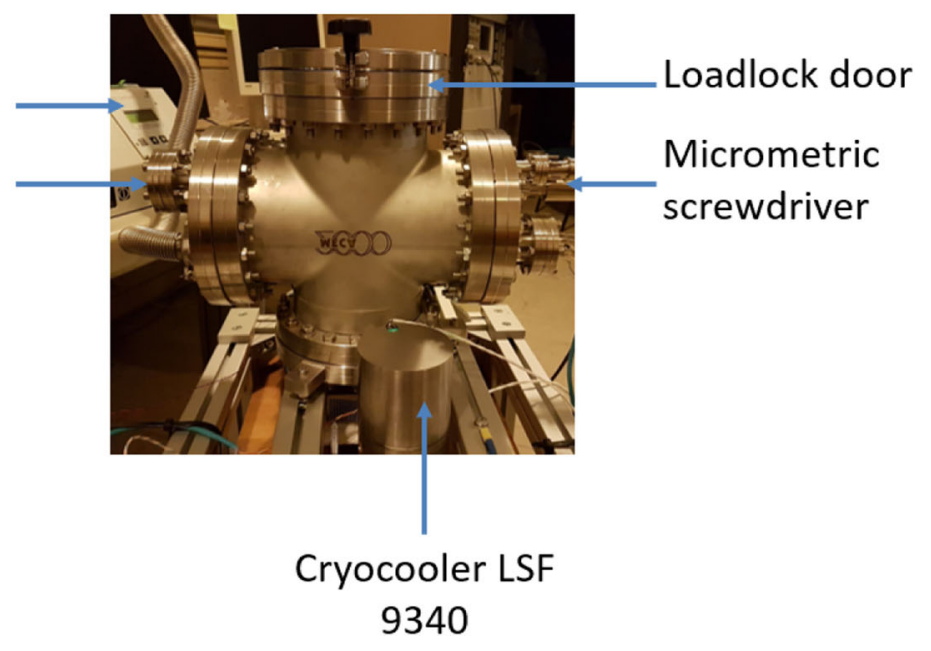

Fig. 1 a Schematic diagram of the experimental setup. b Outside view of the cryostat: the vacuum chamber consists of a sealed four-way cross (see blue outline in Fig. 1a) with four flanges

362-9856, 1/3 B DIN) fixed to the sapphire cylinder near the HTS coil with thermally conductive glue and Kapton ${ }^{\circ}$ tape. The temperature is measured every $10 \mathrm{~ms}$.

In addition, four $100 \Omega$-resistors are placed on the copper plate to provide active warming of the system.

\section{RF measurement system}

Figure 2 shows a diagram of the RF setup, composed of a Keysight E5071C Vector Network Analyzer (VNA) connected to a measurement probe that is inductively 


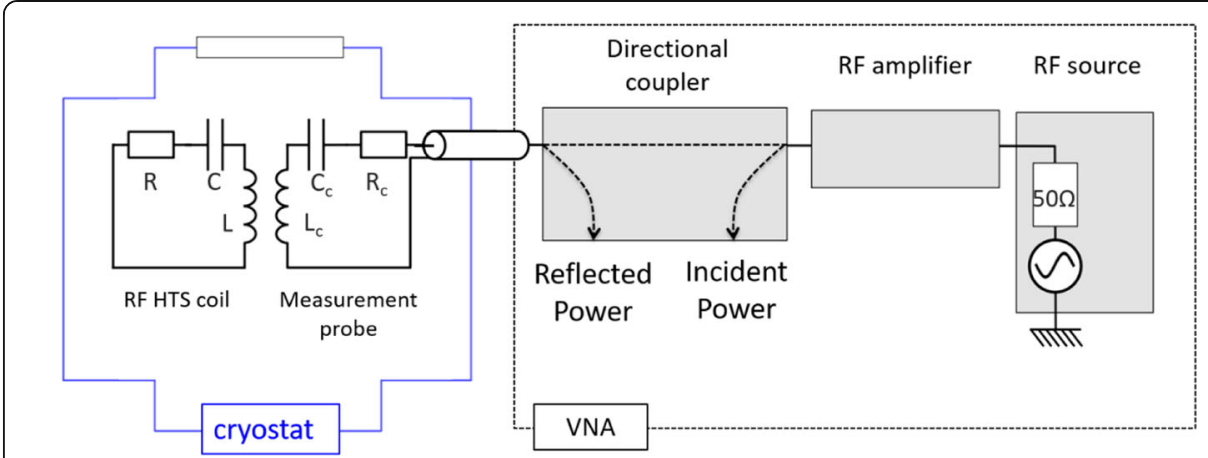

Fig. 2 Scheme of the RF measurement setup. The electrical parameters $\left(Q\right.$ and $f_{0}$ ) of the HTS coil are inductively measured by reflectometry

coupled to the RF coil (Fig. 1). A custom-made Matlab interface was used for VNA control and data acquisition.

A double-sided multi-turn transmission line resonator was used in this study. Each side is composed of 6 turns of $\mathrm{YBa}_{2} \mathrm{Cu}_{3} \mathrm{O}_{7}$ with an average diameter of $12.3 \mathrm{~mm}$, i.e. a total length of $230 \mathrm{~mm}$ and a width of $200 \mu \mathrm{m}$. The superconducting material is deposited on a sapphire dielectric substrate (CERRACO; thickness $=330 \mu \mathrm{m}, \varnothing=11.6 \mathrm{~mm}$ ) and its critical temperature is about $86 \mathrm{~K}$.

RF measurements were performed following the method defined by reference [7]. The electrical parameters of the HTS coil were obtained by measuring its RF response through inductive coupling with a measurement probe and using the single loop probe method [24]. The measurement probe is a $12-\mathrm{mm}$ diameter copper loop, tuned to $f_{0}$ with a $330 \mathrm{pF}$-capacitor and matched using a $50 \Omega$-resistance that can dissipate incident power of up to $10 \mathrm{~W}$.

The single-loop probe method uses a reference data set for the reflection coefficient $\rho_{0}$ measured at $90 \mathrm{~K}$; at this temperature the HTS coil is not resonant. From the reflection coefficient $\rho$, measured in the presence of the coil under test, we can calculate the compensated reflection coefficient, defined as $\rho_{\text {comp }}=\rho-\rho_{0}$ [24]. The HTS coil quality factor $Q$ is obtained from a swept-frequency response analysis of $\rho_{\text {comp }}$, using $Q=f_{0}$ / $[\mathrm{BW}]_{-3 \mathrm{~dB}}$, where $[\mathrm{BW}]_{-3 \mathrm{~dB}}$ is the $3 \mathrm{~dB}$ attenuation bandwidth.

\section{Results}

Figure 3 shows the temperature variation measured by the diode and the Pt100 sensors during the cool-down (a) and warm-up (b) processes. The difference between the temperature measured by the sensors during the cool-down (Fig. 3a) is approximately $2 \mathrm{~K}$ when the temperature of the diode is $70 \mathrm{~K}$. This indicates an imperfect thermal contact between the flat-shaped Pt100 and the cylindrically shaped sapphire cold finger. The precision of the measurement is approximately $3 \mathrm{mK}$ and $37 \mathrm{mK}$ at $70 \mathrm{~K}$ for the diode and for the PT100, respectively. Temperature measured by the diode was chosen as the reference measurement. From room temperature, the system reaches a starting temperature of $70 \mathrm{~K}$ approximately $20 \mathrm{~min}$ after starting the cool-down.

The $100 \Omega$-resistors placed on the copper plate allow a power dissipation of approximately $9 \mathrm{~W}$ across the plate surface. This reduces the time needed to warm the system from $70 \mathrm{~K}$ to $300 \mathrm{~K}$ to $45 \mathrm{~min}$, rather than the $240 \mathrm{~min}$ it originally took (Fig. 3b). 

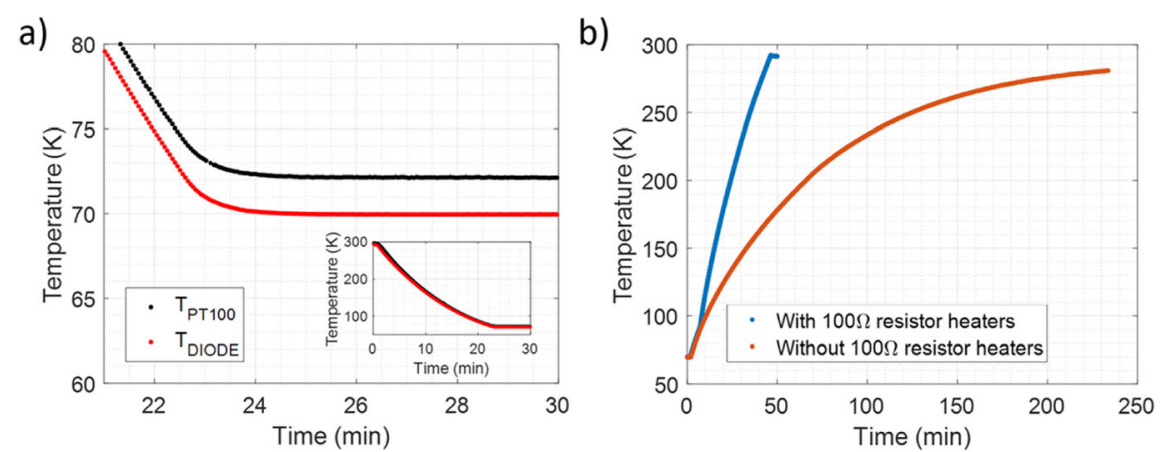

Fig. 3 a The temperature monitored by the two temperature sensors (the diode and the Pt100) is presented as function of time. $\mathbf{b}$ The temperature variation of the system as measured by the diode in the presence and absence of heating elements. The temperature is measured every $10 \mathrm{~ms}$

The RF response, i.e. the $\rho_{\text {comp }}$ modulus of the HTS coil, was measured as a function of frequency for temperatures ranging from 60 to $83 \mathrm{~K}$ at a fixed power of $3 \times 10^{-5} \mathrm{~W}$ (Fig. 4a) and for incident RF power values, varying from $3 \times 10^{-6} \mathrm{~W}$ to $10^{-2} \mathrm{~W}$ at $T=$ $70 \mathrm{~K}$ (Fig. 4b).

A variation of the resonance frequency $\left(f_{O}(60 \mathrm{~K})-f_{O}(76 \mathrm{~K})=0.1 \mathrm{MHz}\right)$ is observed for temperatures between $60 \mathrm{~K}$ and $76 \mathrm{~K}$ (Fig. 4a). For temperatures above $76 \mathrm{~K}$, a widening of the frequency response occurs. A shift of the resonance frequency is also observed due to the change of the quality factor caused by resistance variation. The shape of the frequency response is same for all temperatures. The frequency response is well fitted by a Lorentzian function, allowing the determination of the quality factor of the coil. A $Q$ value of 30,000 was measured at $60 \mathrm{~K}$. Figure $4 \mathrm{~b}$ shows that an increase in power leads to a deformed response of the coil and a decrease in the magnitude of the reflection coefficient. At high incident power $\left(63 \times 10^{-2} \mathrm{~W}\right)$, a slight shift is observed in frequency (approximately $0.01 \mathrm{MHz}$ between $3 \times 10^{-5} \mathrm{~W}$ and $63 \times 10^{-2} \mathrm{~W}$ ), along with a substantial decrease of the reflection coefficient.

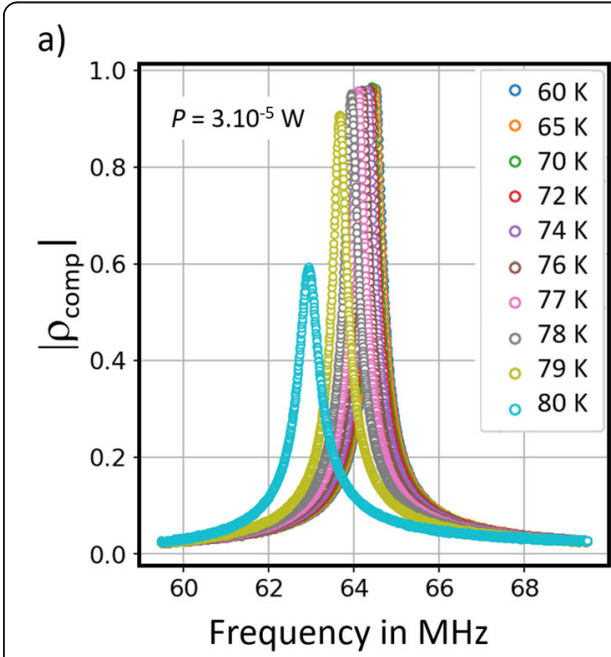

b)

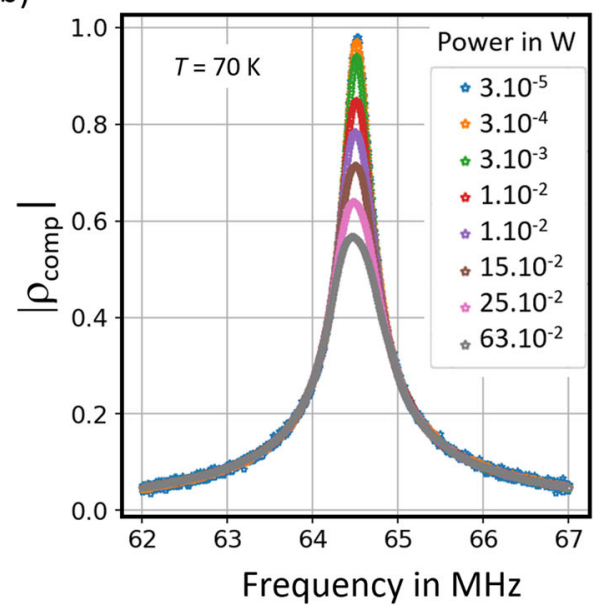

Fig. 4 The RF response of the HTS coil as a function of frequency (a) for different temperatures varying from $60 \mathrm{~K}$ to $83 \mathrm{~K}$ at $3 \times 10^{-5} \mathrm{~W}$, and (b) for different incident power values (from $3 \times 10^{-5} \mathrm{~W}$ to $63 \times 10^{-2} \mathrm{~W}$ ) at $70 \mathrm{~K}$ 
Figure 5 shows the temperature dependence of the normalized $Q$-factor $(\mathrm{Q} / \mathrm{Q}(60 \mathrm{~K}))$ at $3 \times 10^{-5} \mathrm{~W}$, as extracted from the results displayed in Fig. 4 . The dependence of the normalized resonance frequency $f_{0}(T) / f_{0}(60 \mathrm{~K})$ at the same power as function of temperature is displayed (in Fig. 5, circles). At $67 \mathrm{MHz}$ and $77 \mathrm{~K}$, the measured Q-factor was approximately 25,000; the same value was obtained at the same temperature in LN2.

When the temperature increases, we observe a reduction of both the resonance frequency and the quality factor. As the temperature approaches the critical temperature of the HTS material, both Q and $f_{0}$ change more rapidly. This is due to decreases in the superfluid density, which lead to an increase in the equivalent resistance of the coil.

This experimental set-up was used to reproduce an MRI sequence in terms of power level and RF time application. First, an RF pulse with incident power ranging from $10^{-8} \mathrm{~W}$ to $0.6 \mathrm{~W}$ was applied for $11 \mathrm{~ms}$ to mimic the RF pulses used during spin excitation. Two examples of power values, $10^{-3} \mathrm{~W}$ or $0.6 \mathrm{~W}$, are presented in Fig. 6. Next, low power pulses $\left(10^{-5} \mathrm{~W}\right)$ were applied for $8 \mathrm{~ms}$ to mimic the NMR signal amplitude during reception. The switching between high and low resistance values occurs on a time shorter than the minimum time resolution of the network analyzer which is $12 \mu \mathrm{s}$. The resistance $R$ of the HTS coil was extracted from the measured $\rho_{\text {comp }}$ using the method presented in [7]. Figure 7 shows the HTS coil surface resistance $R_{s}$ (in $\mathrm{m} \Omega /$ $\mathrm{mm}^{2}$ ) values as a function of the RF incident power. The resistance value $R_{s}$ can be decreased from over $400 \mu \Omega / \mathrm{mm}^{2}$ to $200 \mu \Omega / \mathrm{mm}^{2}$ in less than $12 \mu \mathrm{s}$.

\section{Discussion}

This cryostat is designed to accommodate different configurations and sizes of HTS coils, and enables users to control vacuum, temperature and power output throughout the experiment. It also ensures a short time to cool and heat the system (20 and $45 \mathrm{~min}$ respectively) compared to the time required for other types of cryogen-free cryostats, which can be in excess of $5 \mathrm{~h}[25,26]$. This rapidity makes it possible to evaluate several sensors in a short time. Temperature measurement is provided by the diode with a precision of approximately $3 \mathrm{mK}$ at $70 \mathrm{~K}$. The power dissipation in the Pt100 sensor

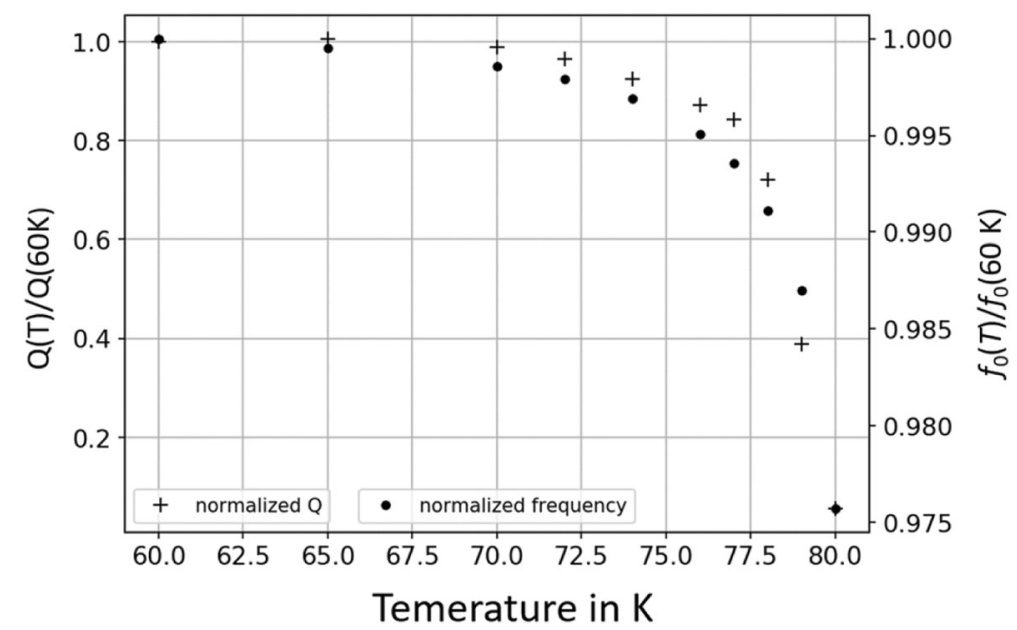

Fig. 5 Dependence of the normalized Q-factor (cross marker) and of the normalized resonance frequency $f_{0}(T) / f_{0}(60 K)$ (circles) as a function of the temperature probed by the diode 


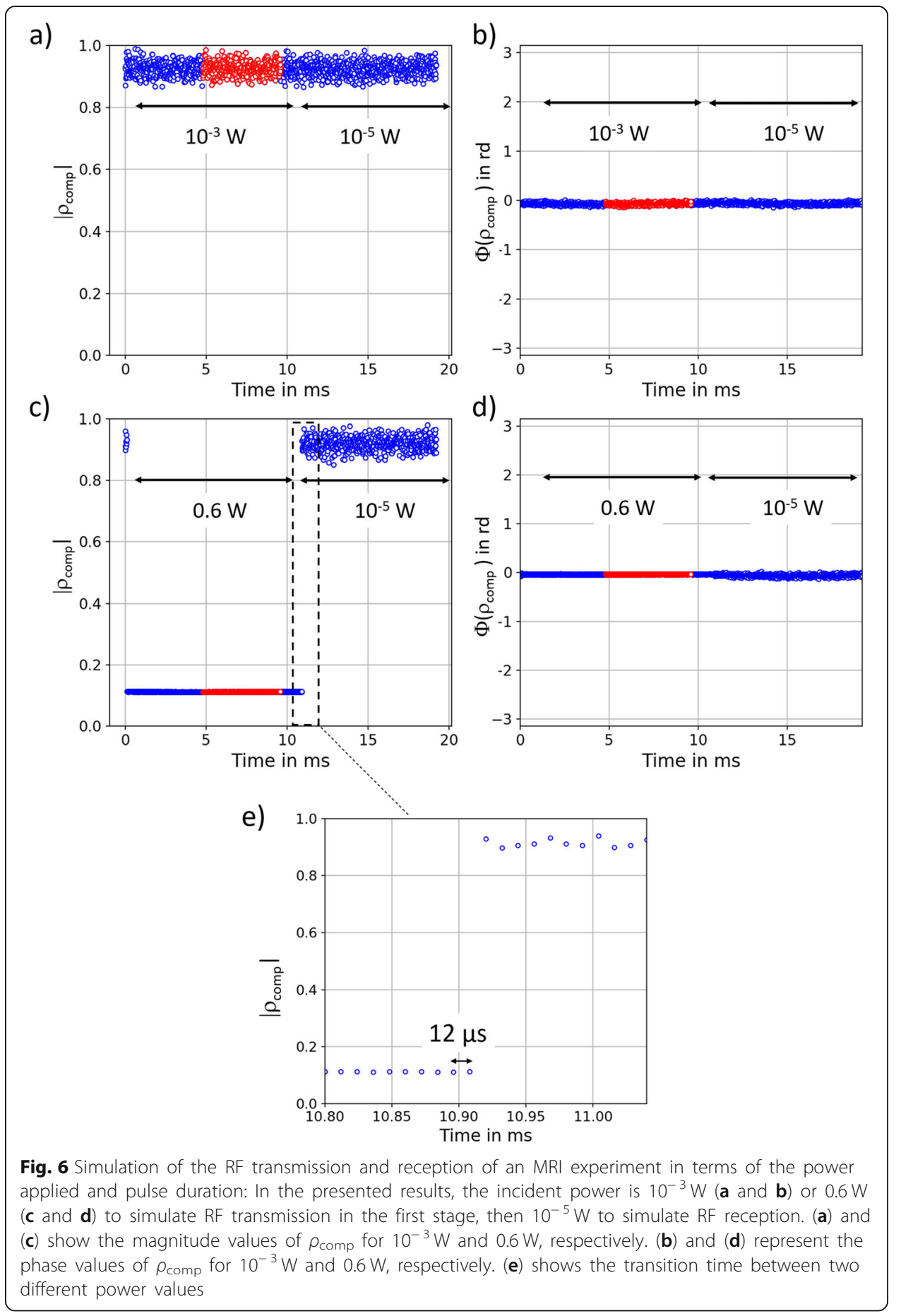

caused Joule heating that amounted to a dissipation of $1.5 \times 10^{-5} \mathrm{~W}$, while the diode sensor dissipated $10^{-3} \mathrm{~W}$. A minimum cryocooler cooling power of $4 \mathrm{~W}$ is required to achieve a cold tip temperature of $60 \mathrm{~K}$. It can be assumed that Joule heating from the temperature sensors is negligible.

The observed behavior of the normalized $f_{0}(T)\left(f_{0}(T) / f_{0}(60 \mathrm{~K})\right)$ and $Q$-factor $(Q(T) /$ $Q(60 \mathrm{~K}))$ is similar to previous measurements reported in [19] using a similar coil with an LN2 cryostat combined with a vacuum pump. Nevertheless, the latter technique is 


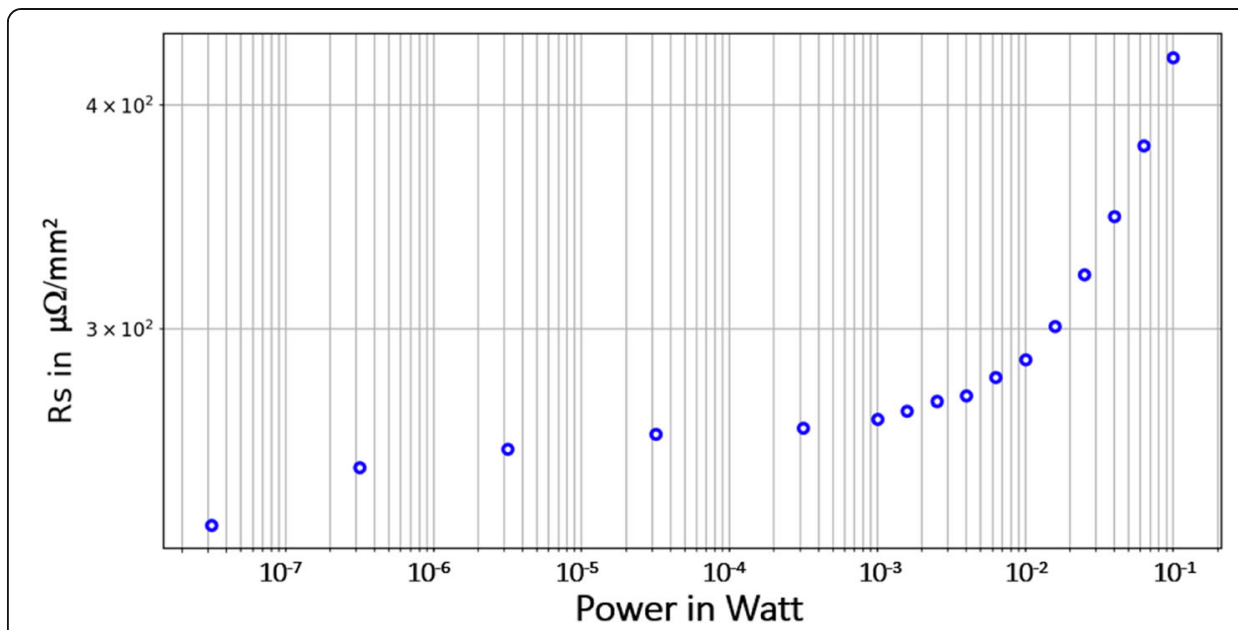

Fig. 7 Coil surface resistance was measured from the average of $\rho_{\text {comp }}$ values (see red circles shown in Fig. 6) as function of the different incident power investigated

not easy to implement and makes difficult to obtain a stable temperature. This makes the electrical behavior of the HTS coil unstable, which has electrical properties that are temperature dependent.

The measurements of the resonance frequency as a function of temperature (Fig. 5) show that the HTS coil could be fine-tuned through temperature control [27]. This method can replace previously reported tuning methods for HTS coils that present several disadvantages. Tuning by inductive coupling of HTS coil to a closed copper loop [15] may degrade the quality factor of the HTS coil and lead to an increase of its resonance frequency [28]. Tuning by placing a dielectric pad on the surface of the HTS coil can only decrease the resonance frequency [29], and may also cause additional losses, depending on the dielectric material used.

The extracted $R_{\mathrm{s}}$ values at $64 \mathrm{MHz}$ support the results presented in [5] at $100 \mathrm{MHz}$. This study found that the transition between two different values of $R_{\mathrm{s}}$, corresponding to low and high incident power level, is achieved in less than $12 \mu \mathrm{s}$, which is two orders of magnitude lower than the transition time between RF transmission and reception steps in MRI sequences. This is could be used to passively decouple the HTS coil during RF transmission (high incident power involved), yet use the coil during RF reception (low incident power involved) [30]. Thus, homogeneous excitation could be achieved by using a separate volume coil for RF transmission. Finally, this system permits the study of different resonator geometries to identify the most suitable designs to sufficiently increase $R_{\mathrm{s}}$ at high power level, while maintaining a low $R_{\mathrm{s}}$ at low power level.

\section{Conclusion}

This paper describes the development and validation of a custom-made low-cost cryostat for the characterization of the nonlinear response of HTS coils as a function of incident RF power, at working temperatures ranging between 60 and $300 \mathrm{~K}$. The determination of HTS coil RF properties is essential to understand coil behavior during MRI experiments. We have demonstrated that the resistance $R_{\mathrm{s}}$ of the HTS coil changes as a function of the incident power level, and the transition time is achieved in 
less than $12 \mu$ s. This may be used as a passive decoupling method for HTS coil. Future work will focus on the combination of RF measurement and magneto-optical imaging [31] to access the macroscopic (i.e. EM properties) and microscopic properties of the HTS coil. This combination will allow for the in-depth characterization of the performance of HTS coils for a better understanding of the nonlinear behavior of HTS resistivity. The loadlock door of the cryostat presented in Fig. 1 allows for optical access, and is therefore compatible with a magneto-optical set-up. Furthermore, the geometry of the cryostat provides sufficient room for multiple HTS coils or large array of coils [32] and makes it possible to implement an automated matching system for a reliable and a precise adjustment of the coil matching [29].

\section{Abbreviations}

HTS: High-Temperature Superconducting; RF: Radio Frequency; MRI: Magnetic Resonance Imaging; SNR: Signal-toNoise Ratio; EM: Electromagnetic; NMR: Nuclear Magnetic Resonance; Q: Quality factor; LN2: Liquid Nitrogen; J-T: JouleThomson; VNA: Vector Network Analyzer

\section{Acknowledgements}

Authors would like to thank the Île de France Region in the framework of DIM Nano-K and the French Agence Nationale de la Recherche (ANR) for funding the project.

\section{Authors' contributions}

All authors read and approved the final manuscript.

\section{Funding}

This work has been supported by the Île de France Region in the framework of DIM Nano-K and by the French Agence Nationale de la Recherche (ANR) under grant No. ANR-14-CE17-0003 (SupraSense project).

\section{Availability of data and materials}

The datasets generated and/or analysed during the current study are not publicly available due to the policy of the participating laboratories but are available from the corresponding author on reasonable request.

\section{Competing interests}

The authors declare that they have no competing interests.

\section{Author details}

${ }^{1}$ Université Paris-Saclay, CEA, CNRS, Inserm, BioMaps, Orsay, France. ${ }^{2}$ Unité Mixte de Physique, CNRS, Thales, Université Paris-Saclay, 91767 Palaiseau, France. ${ }^{3}$ Laboratoire des Solides Irradiés, Ecole polytechnique, CNRS, CEA, Université Paris-Saclay, Palaiseau, France. ${ }^{4}$ Centre de Nanosciences et de Nanotechnologies, CNRS, Université Paris-Saclay, Palaiseau, France. ${ }^{5}$ Irfu, CEA Paris-Saclay, Université Paris-Saclay, F-91191 Gif-sur-Yvette, France.

Received: 4 November 2019 Accepted: 2 July 2020

Published online: 29 July 2020

\section{References}

1. Sanchez-Heredia JD, Szocska Hansen ES, Laustsen C, Zhurbenko V, Ardenkjær-Larsen JH. Low-noise active decoupling circuit and its application to $13 \mathrm{C}$ cryogenic RF coils at 3 T. Tomography. 2017;3(1):60-6.

2. Darrasse L, Ginefri JC. Perspectives with cryogenic RF probes in biomedical MRI. Biochimie. 2003;85:915-37.

3. Laistler $E$. Magnetic resonance micro-imaging of the human skin in vivo using miniature radio frequency coils; 2010.

4. Poirier-Quinot M, Ginefri J-C, Girard O, Robert P, Darrasse L. Performance of a miniature high-temperature superconducting (HTS) surface coil for in vivo microimaging of the mouse in a standard 1.5T clinical whole-body scanner. Magn Reson Med. 2008;60(4):917-27.

5. Shen Z-Y. High-temperature superconducting microwave circuits [internet]. Artech house; 1994. p. 272. Available from: https://us.artechhouse.com/High-Temperature-Superconducting-Microwave-Circuits-P870.aspx.

6. Anlage SM, Hu W, Vlahacos CP, Steinhauer D, Feenstra BJ, Dutta SK, et al. Microwave nonlinearities in high-Tc superconductors: the truth is out there. J Supercond. 1999 Apr 1;12(2):353-62.

7. Girard O, Ginefri J-C, Poirier-Quinot M, Darrasse L. Method for nonlinear characterization of radio frequency coils made of high temperature superconducting material in view of magnetic resonance imaging applications. Rev Sci Instrum. 2007;78(12):124703.

8. Hoult DI, Richards RE. The signal-to-noise ratio of the nuclear magnetic resonance experiment. J Magn Reson. 1976;24: $71-85$.

9. Hoult DI, Richards RE. The signal-to-noise ratio of the nuclear magnetic resonance experiment. J Magn Reson 1969. 1976;24(1):71-85.

10. Hall AS, Nm A, Button TW, Gilderdale DJ, Gehring KA, Young IR. Use of high temperature superconductor in a receiver coil for magnetic resonance imaging. Magn Reson Med. 1991;20(2):340-3.

11. Penn SJ, Alford NM, Bracanovic D, Esmail AA, Scott V, Button TW. Thick film YBCO receive coils for very low field MRI. IEEE Trans Appl Supercond. 1999 Jun;9(2):3070-3. 
12. van Heteren JG, James TW, Bourne LC. Thin film high temperature superconducting RF coils for low field MRI. Magn Reson Med. 1994;32(3):396-400.

13. Wosik J, Nesteruk K, Xie L-M, Strikovski M, Wang F. MillerJr. JH, et al. high-Tc superconducting rf receiver coils for magnetic resonance imaging of small animals. Phys C Supercond. 2000;341-348(4):2561-4.

14. Wright AC, Song HK, Wehrli FW. In vivo MR micro imaging with conventional radiofrequency coils cooled to $77^{\circ} \mathrm{K}$. Magn Reson Med. 2000;43(2):163-9.

15. Ginefri J-C, Darrasse L, Crozat P. High-temperature superconducting surface coil for in vivo microimaging of the human skin. Magn Reson Med. 2001;45(3):376-82.

16. Mann LD, Duerk JL, Wendt M, Hansen KL, Brey WW. A small superconductive surface coil with enhanced bandwidth and active decoupling; 1998. p. 435.

17. Miller JR, Hurlston SE, Ma QY, Face DW, Kountz DJ, MacFall JR, et al. Performance of a high-temperature superconducting probe for in vivo microscopy at 2.0 T. Magn Reson Med. 1999;41(1):72-9.

18. Odoj F, Rommel E, Kienlin M, Haase A. A superconducting probehead applicable for nuclear magnetic resonance microscopy at 7 T. Rev Sci Instrum. 1998;69(7):2708-12.

19. Lambert S, Ginefri J-C, Poirier-Quinot M, Darrasse L. High-temperature superconducting radiofrequency probe for magnetic resonance imaging applications operated below ambient pressure in a simple liquid-nitrogen cryostat. Rev Sci Instrum. 2013;84(5):054701.

20. Wright AC, Song HK, Elliott DM, Wehrli FW. Use of a joule-Thomson micro-refrigerator to cool a radio-frequency coil for magnetic resonance microscopy. Rev Sci Instrum. 2004;76(1):014301.

21. Hill HDW. Improved sensitivity of NMR spectroscopy probes by use of high-temperature superconductive detection coils. IEEE Trans Appl Supercond. 1997;7(2):3750-5.

22. Thompson PS, Ackermann RA, Hedeen RA. A two-stage pulse tube cryo-cooled MRI magnet. AIP Conf Proc. 2002;613(1): 649-53.

23. Radebaugh R. Cryocoolers: the state of the art and recent developments. J Phys Condens Matter Inst Phys J. 2009; 21(16):164219.

24. Ginefri J-C, Durand E, Darrasse L. Quick measurement of nuclear magnetic resonance coil sensitivity with a single-loop probe. Rev Sci Instrum. 1999;70(12):4730-1.

25. Authelet G, Bonelli A, Poirier-Quinot M, Ginefri J-C, Saniour I, Baudouy B. All polymer cryogen free cryostat for \$lupmu\$MRI application at clinical field. IOP Conf Ser Mater Sci Eng. 2019 Apr;502:012156.

26. Authelet G, Poirier-Quinot M, Ginefri J-C, Bonelli A, Baudouy B. Conceptual design of a cryogen-free $\mu M R I$ device. IOP Conf Ser Mater Sci Eng. 2017;278(1):012122.

27. Saniour I, Authelet G, Baudouy B, Dubuisson R-M, Jourdain L, Willoquet G, et al. A temperature-controlled cryogen free cryostat integrated with transceiver-mode superconducting coil for high-resolution magnetic resonance imaging. Rev Sci Instrum. 2020;91(5):055106.

28. Saniour I, Authelet G, Baudouy B, Dubuisson R-M, Jourdain L, Willoquet G, et al. Performance of a novel cryogen-free cryostat with an automated temperature control for fine tuning of High Temperature Superconducting RF coils: highresolution MR imaging at 1.5 T. In ISMRM; 2019. p. 0570.

29. Li Z, Bou Abboud G, Guillot G, Hosseinnezhadian S, Jourdain L, Poirier-quinot M, et al. Study of two contact-less tuning principles for small monolithic radiofrequency MRI coils and development of an automated system based on piezoelectric motor. Sens Actuators Phys. 2016;241:176-89.

30. Saniour I, Authelet G, Baudouy B, Dubuisson R-M, van der Beek CJ, Darrasse L, et al. Novel passive decoupling approach for high resolution HTS RF coils based on the nonlinear electrical properties of superconductors. Rotterdam: ESMRMB 2019, 36th Annual Scientific Meeting. Springer; 2019. https://doi.org/10.1007/s10334-019-00754-2.

31. Geahel M, Jouanny I, Gorse-Pomonti D, Poirier-Quinot M, Briatico J, van der Beek CJ. Edge contamination, bulk disorder, flux front roughening, and multiscaling in type II superconducting thin films. Condens Matter. 2017;2(3):27.

32. Wosik J, Xue L, Xie L-M, Kamel MR, Nesteruk K, Bankson JA. Superconducting array for high-field magnetic resonance imaging. Appl Phys Lett. 2007;91(18):183503.

\section{Publisher's Note}

Springer Nature remains neutral with regard to jurisdictional claims in published maps and institutional affiliations.

\section{Submit your manuscript to a SpringerOpen ${ }^{\circ}$ journal and benefit from:}

- Convenient online submission

- Rigorous peer review

- Open access: articles freely available online

High visibility within the field

- Retaining the copyright to your article

Submit your next manuscript at $\boldsymbol{\nabla}$ springeropen.com 